\title{
Modeling of Electric Railway Vehicle for Harmonic Analysis of Traction Power-supply System using Spline Interpolation in Frequency Domain
}

\author{
$\begin{array}{lll}\text { K.H. Yuen } & \text { M.H. Pong L. Lo Z.M.Ye }\end{array}$ \\ Department of Electrical \& Electronic Engineering \\ The University of Hong Kong \\ Pokfulam Road, Hong Kong, PRC
}

\begin{abstract}
It is essential to model nonlinear traction converter loads for harmonic analysis of traction systems. A behavioral model in frequency domain to represent electric railway vehicle based on testing and measurement is proposed for harmonic analysis. The harmonic current characteristics are represented by a set of polynomials generated from cubic smoothing spline interpolation. The purpose of this paper is to report and discuss the development of an electric railway model for harmonic analysis and demonstrate results from the simulation with this train load model. System simulation based on this model is performed and the results match satisfactorily with field measurement.
\end{abstract}

\section{INTRODUCTION}

In recent years, with the popularization of power electronics equipment in railway traction applications such as converter-driven motor drives, battery chargers and auxiliary power supplies, harmonic pollution has become more serious, thus giving adverse effects to power-supply systems. Thus, railway engineers pay more attention to power quality of traction power-supply systems. Harmonic contents in traction currents are one of the most important aspects of power quality. Various methods to deal with such problems were carried out [2][3]. A load current model to represent electric railway vehicles is required for harmonic analysis of traction systems. Pan Yinxuan, Sun Shugin, Li Chunhua [4] used a circuit model for a phase-controlled thyristor vehicle with idealized parameters to simulate traction loads. J. Shen, J.A. Taufiq, A.D. Mansell [5] given analytical solution to harmonic characterisitcs of traction PWM converters. In our analysis, instead of using an exact analytical model, a behavioral model in frequency domain to represent electric railway vehicle based on testing and measurement is proposed. The model consists of traction drive loads and other auxiliary loads. This method provides an alternative which requires measurements and general operations of the traction drive only. Hence, one of the advantages of this modelling technique is eliminating the requirement of knowing the detail design, parameters and control logic of traction drives. Railway companies who may not know all these in detail can perform testing and use the measurements to build a model for analysis at system level. In this paper, the development of an electric railway load model for harmonic analysis is presented. Simulation results with this load model will be shown as well.

\section{HARMONIC Distortions}

Nonlinear and switch loads generate a lot of harmonic distortion to the voltage and current. It is essential to determine the level of harmonics. In an electrical system, total harmonic distortion current can be used to indicate the harmonic levels.

$$
I_{T H D}=\sqrt{\sum_{h \neq 1} I_{h}^{2}}
$$

However, it is not easy to compare the harmonic levels of two systems of different power rating. In order to evaluate and compare the levels of harmonics, the most commonly used measure of harmonic distortions is called total harmonic distortion (THD) factor.

$T H D=\sqrt{\sum_{h \neq 1} I_{h}^{2}} / I_{1}$

\section{Principle of Electric Railway Vehicle Modeling}

In many modern electric railway, most harmonic currents are generated by thyristor-converter of electric railway vehicle. A simplified circuit diagram for the thyristor-controlled railway vehicle is shown in Fig. 1. Therefore, modeling of electric railway vehicle is indispensable for harmonic evaluation. Accurate parameters should be required to build an analytical circuit model. However, such approach cannot conveniently give accurate results due to the fact that many parameters are unknown, especially with existing railway vehicles whereas computations required to solve circuit equations are heavy.

A behavioral modeling technique is proposed. A train is treated as a black box. It is assumed that the behavior of traction converter of railway vehicles is prewise-linear for each operation notch. By applying cubic curve-smoothing 
technique on interpolation of measurement, a rather simple model for railway vehicle is obtained. Because the model is obtained from real-world measured data, it is a good approximation to a real train. Measurements for the analysis were taken from both electric motoring unit (EMU) and power-supply substation. Current drawn by EMU at different train speeds and notch settings forms the basis of the model.

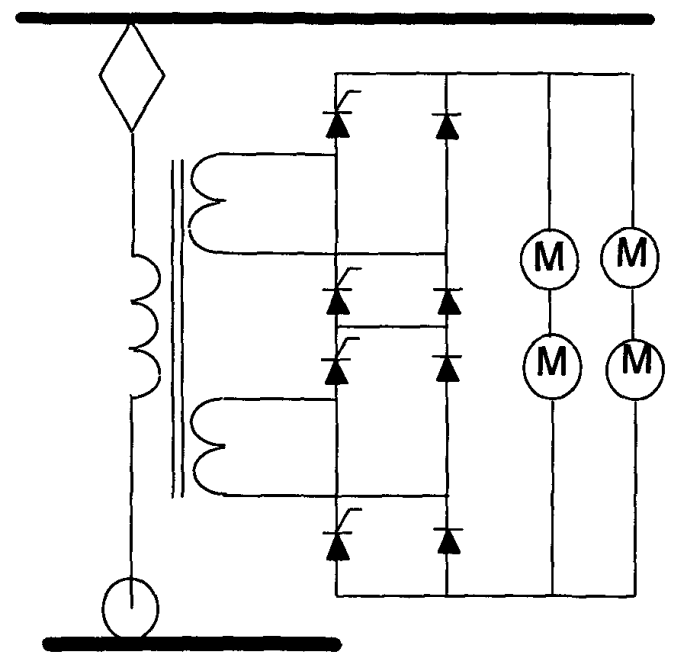

Fig. 1 A simpified circuit for thyristor-controlled drive

Tests for train characteristic were performed at various conditions for the actual system. Measured waveforms at various speeds and notches are converted into frequency domain by Discrete Fourier Transform algorithm. Hence, all waveforms are represented in frequency domain.

Measurements are discrete. Hence, interpolation is required to achieve continuity of the characteristic function of railway vehicle at each harmonic frequency. Cubic smoothing spline interpolation technique [1] is used. The harmonic current characteristics are calculated from a set of polynomials generated by cubic spline smoothing interpolation. Hence, prewise-linear polynomials are employed to represent the mapping curves of harmonic currents. Changes in converter current waveform generated by supply voltage distortion are neglected for simplicity of the analysis. A polynomial is used between two adjacent measured states. Fundamental and harmonic currents at different speeds and notches are determined by a set of polynomials. The degree of these polynomials is 3 .

Cubic smoothing spline interpolation is better than linear interpolation because the characteristic of converter with continuous varying fire angle can be assumed to be smooth.
Moreover, interpolation in frequency domain is reasonable for harmonic analysis and the whole system is analyzed in frequency domain.

The word "smoothest" means the following measure of roughness which is minimized as below

$$
F\left(D^{m} f\right)=\int\left(D^{m} f\right)^{2} d x
$$

where $D^{m}$ refers $m$-th derivative. The value of $m$ is 2 for cubic smoothing spline. Furthermore, the distance of the function $f$ from the given data is measured by

$E(f)=\Sigma w(j)(y(i)-f(x(j)))^{2}$

The cubic smoothing spline function $f$ to the given data sets $x$, and $y$ is constructed for the specific smoothing parameter $p$ where $p \in[0,1]$ and the specified weight vector $w$. The smoothing spline minimizes the expression:

$p \Sigma_{i} w(i)\left((y(i)-f(x(i)))^{2}+(1-p) \int\left(D^{2} f\right)^{2} \mathrm{~d} x\right.$

with all values of the weighting vector $w$ are chosen as one. This implies

$\min \left[p \Sigma_{i}\left((y(i)-f(x(i)))^{2}+(1-p) \int\left(D^{2} \mathrm{f}\right)^{2} \mathrm{~d} x\right]\right.$

For $p=0, \mathrm{f}$ is the least-squares straight line fit to data. For $p=1, f$ becomes the variational cubic spline interpolant. The value of $p$ should be selected according to the smoothing of the characteristics of the curve. A set of polynomials is used in mapping function of spline where $m$ is the number of the measured states:

$f(x)=$

$$
\begin{aligned}
& k_{3}(1) x^{3}+k_{2}(1) x^{2}+k_{1}(1) x+k_{0}(1) x, x \in[x(0), x(1)] \\
& k_{3}(2) x^{3}+k_{2}(2) x^{2}+k_{1}(2) x+k_{0}(2) x, x \in(x(1), x(2)] \\
& \ldots \ldots \\
& k_{3}(m) x^{3}+k_{2}(m) x^{2}+k_{1}(m) x+k_{0}(m) x, x \in(x(m-1), x(m)]
\end{aligned}
$$

In our analysis, $f$ represents a function of harmonic current at a notch with input variable $x$ which refers to the train speed. The characteristics of fundamental and harmonic currents can be acquired by measurements from a typical EMU. Plots of fundamental, third and fifth harmonic currents against speed are shown in Fig. 2. The total harmonic distortion current $\left(I_{T H D}=\sqrt{\Sigma_{h \neq 1} I_{h}^{2}}\right)$ is also plotted in Fig. 3 and Fig. 4. The time domain waveforms can be restored by 
applying Inverse Discrete Fourier Transform algorithm to convert simulated results in time domain.
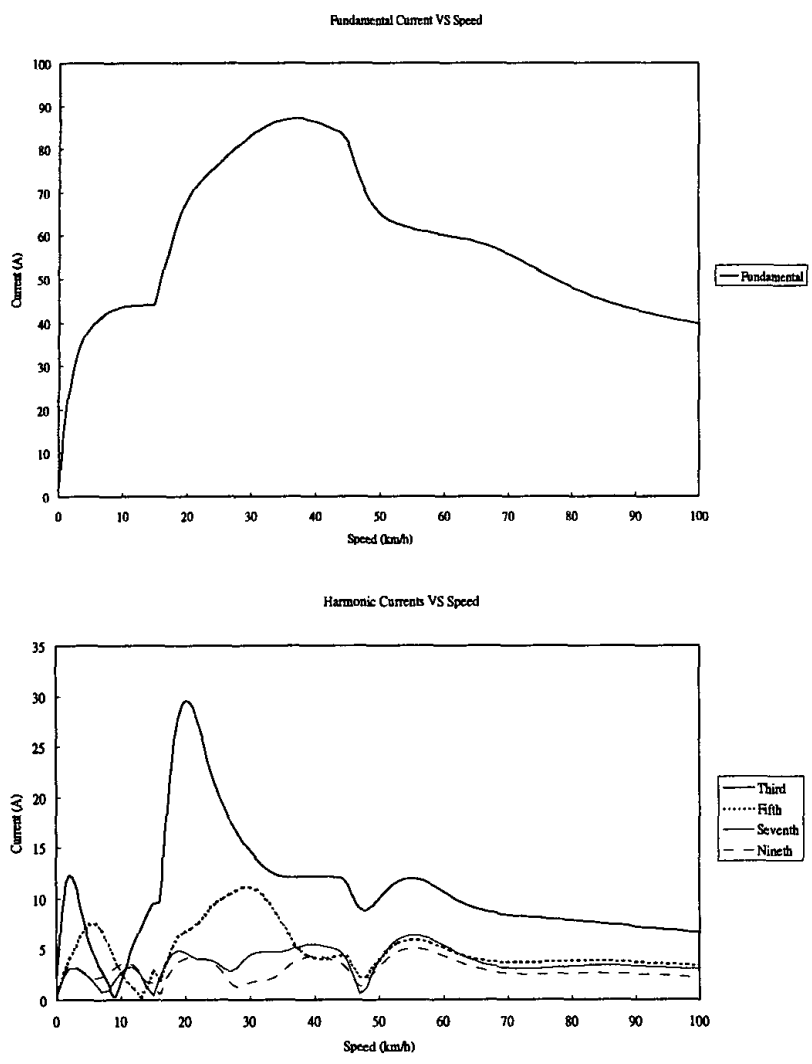

Fig. 2 Characteristics of current Vs speed

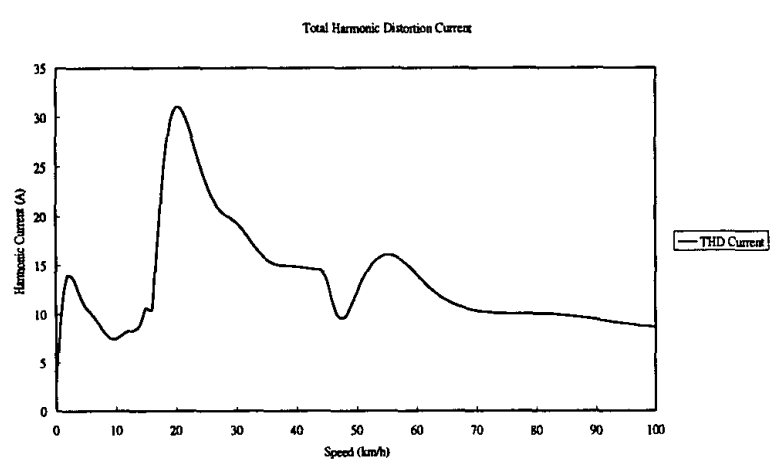

Fig. 3 Total harmonic distortion current Vs speed

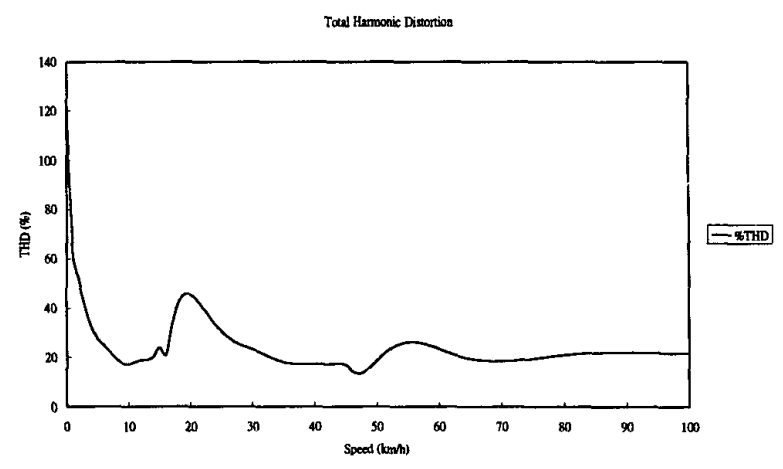

Fig. 4 Total harmonic distortion factor Vs speed

\section{INTEGRation OF TRAin Model to Traction POWER-SUPPLY SYSTEM}

The overall network is modeled in frequency domain. Harmonic flows can be calculated for each harmonic frequency. EMUs are represented as current sinks in various frequency as discussed above. In our case, the length of overhead feeders is less than $10 \mathrm{~km}$. Hence, a short line model is used for the overhead feeder. Substation includes both transformer and capacitor bank for compensating reactive power and also suppressing third harmonics because the capacitor banks are tuned with series inductor to provide suppression on third harmonics. Integration of the train model into other component models forms the traction power-supply system used in studying the power quality as in Fig. 5.
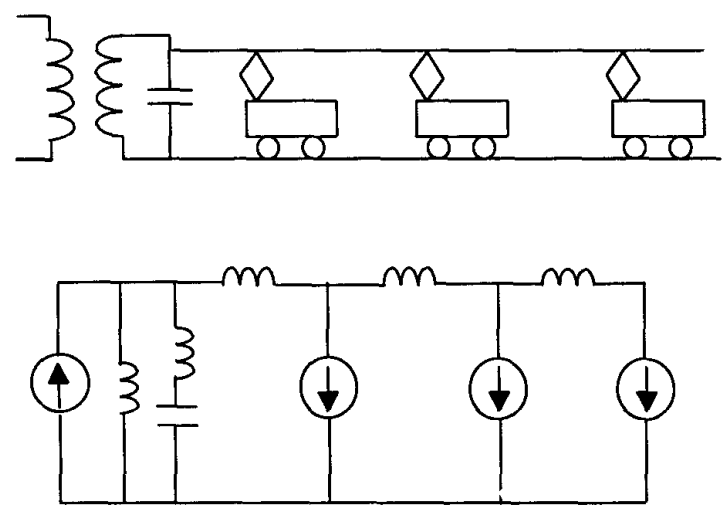

Fig. 5 Model of Traction Power-supply System 
The capacitor bank is simulated as an impedance ( $j X_{L} h$ $\left.j X_{C} / h\right)$ ohm at different frequencies where $h$ is the harmonic number, $X_{l}$ is the inductance of cap bank and $X_{c}$ is the capacitance of cap bank.

In our case, a power substation feeds more than one overhead line. Fig. 6 shows the AC overhead rail system being studied. There are two power substation located at two sites. Between the two power substations, there is a neutral section point.

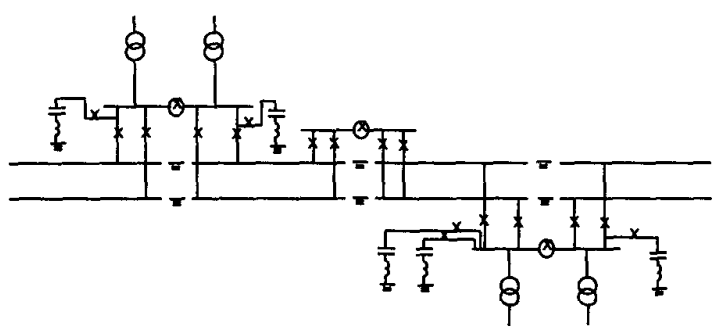

Fig. 6 Overall Traction Power-supply System

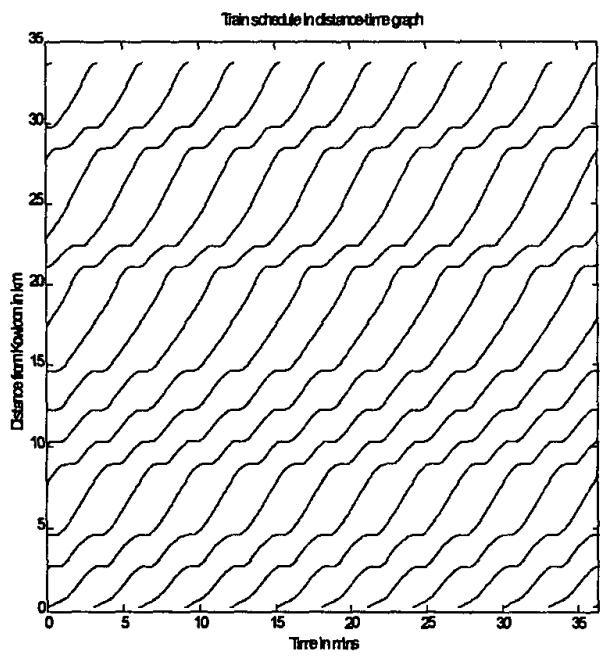

Fig. 7 Train distance-time graph

The time profiles of train speed and position are considered as input variables. Fig. 7 shows a distance-time graph for multi-train operation profile with identical speed-distance relation as in Fig. 8. The simulation is performed at discrete time step. At each time step, a snapshot of the traction network and all train operating conditions are calculated based on the train profile. Substation voltage and current, capbank current can then be computed.

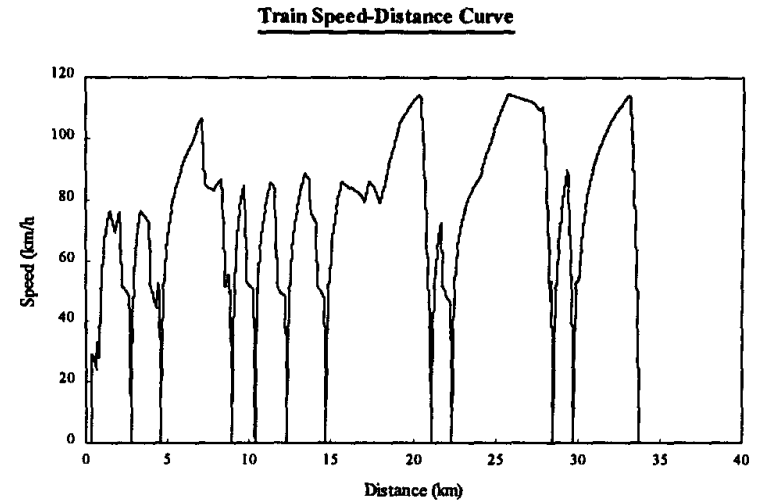

Fig. 8 Train speed-distance profile

\section{Results}

With a given train profile, the train model can be applied to obtain the variations of power factor and harmonic distortion of the overall system for a fixed time interval and results will be obtained. Here some of results about the train model using our proposed modeling technique are shown.

The followings are some of the measured waveforms of substation voltage and current before passing through a 2MVA capacitor bank.
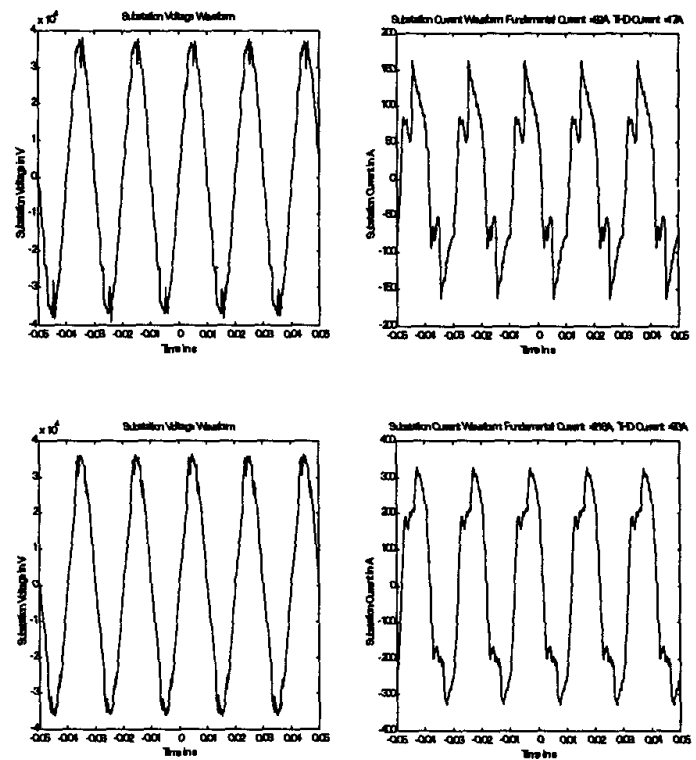

Fig. 9 Measured substation voltage and current waveforms 
Fig. 10 and Fig. 11 show the simulated substation voltage and current waveforms for a train running at low speed range. Comparing Fig. 10 an Fig. 11 with Fig. 9, the simulated results of substation voltage and current with a 2MVA capacitor bank are very close to the actual measurement.
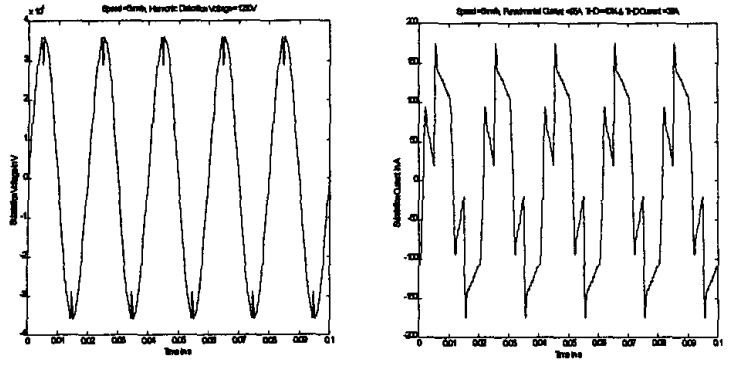

Fig. 10 Simulated substation voltage and current for a train at $5 \mathrm{~km} / \mathrm{h}$
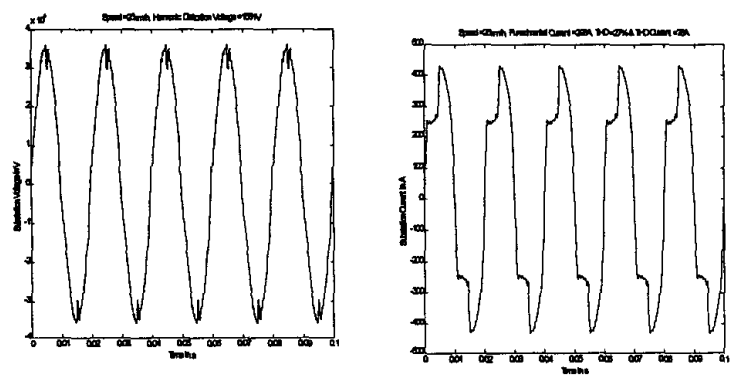

Fig. 11 Simulated substation voltage and current for a train at $25 \mathrm{~km} / \mathrm{h}$

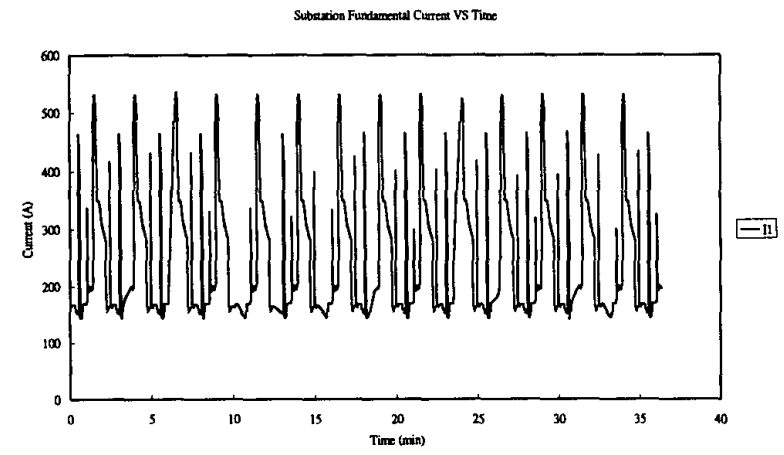

Fig. 12 Simulated substation fundamental current Vs time

This model is integrated to the traction power supply system as discussed in previous section. The substation fundamental current against time for multi-train case is plotted in Fig. 12 and its low-order harmonic currents are shown in Fig. 13

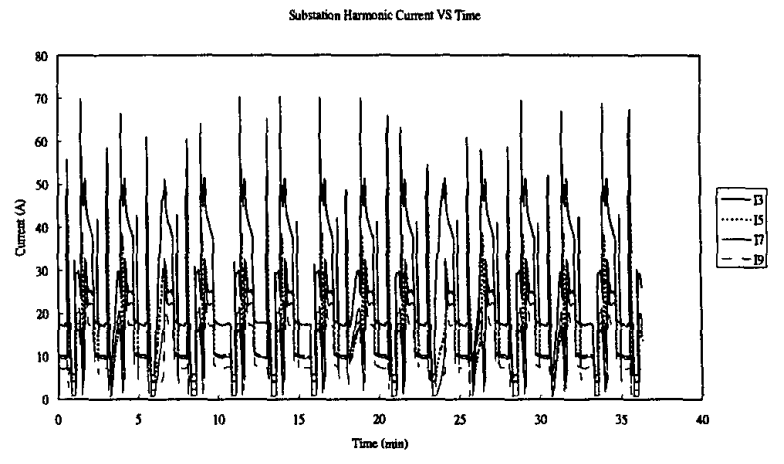

Fig. 13 Simulated substation harmonic currents Vs time for multi-train operation

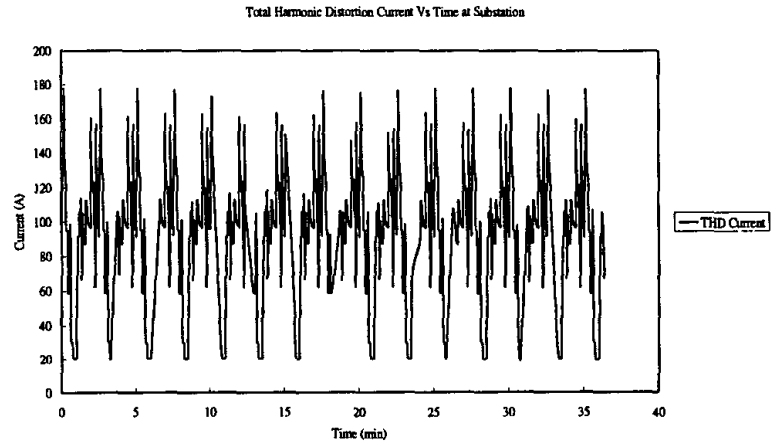

Fig. 14 Simulated THD Current Vs time at substation for muti-train operation

The variations of fundamental and harmonic currents change quite large due to the operation of trains according to the train schedule.

Fig. 14 and Fig. 15 show the results of harmonic distortion of the substation and Fig. 16 presents the variation of power factor against time.

The above results demonstrate the integration of the proposed electric railway vehicle model to the traction power supply system. The results agree with the actual situation that train operation governs the variations of traction loads in the electric railway system. 


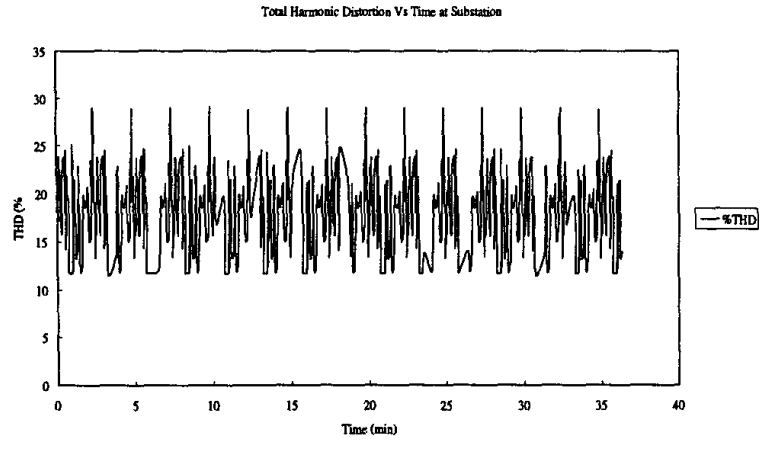

Fig. 15 Simulated \%THD factor Vs time at substation for multi-train operation

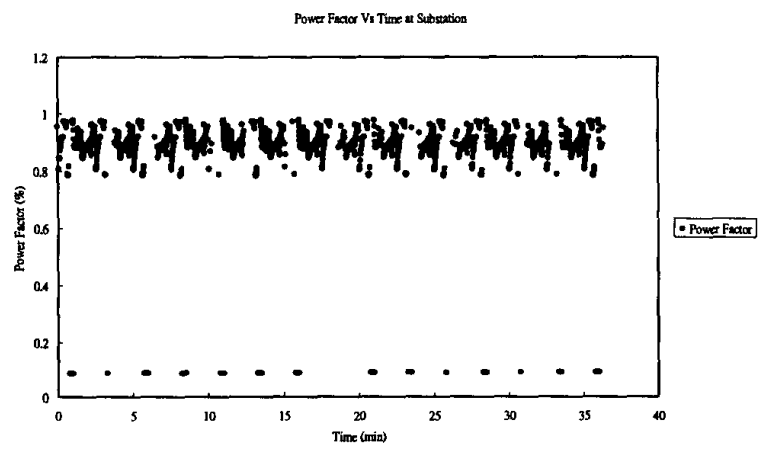

Fig. 16 Simulated power factor Vs time at substation for multi-train operation

\section{CONCLUSIONS}

A new modeling method for electric railway vehicle is proposed. A railway traction system which integrated with the model is simulated with this method. To establish this model, detail information on converter design, accurate electrical parameters and control algorithm of traction drive can be avoided while it gives a good approximation to the real system. The train model performs well in low frequency harmonics such as third, fifth and seventh. For higher harmonics, the model represents these in average sense which is appropriate for power harmonic studies. With the system model, power factor and harmonics are studied.

\section{ACKNOWLEDGMENT}

The authors acknowledge Mr. Tony Lee, Rolling Stock Design Manager, Mr. Lam, High voltage Engineering
Manager and their colleges from East Rail Division, Kwoloon-Cantoon Railway Corporation for their help and arrangement of our measurement and testing.

\section{REFERENCE}

[1] Handbook on SPLINES for the user Eugene V. Shikin, Alexander I. Plis

[2] Time domain analysis of harmonic interaction between traction vehicle and DC supply A. M. Walczyna, P. Janiszewski, Electric Railways in a United Europe, 27-30 March 1995 Conference

[3] Modelling of traction load distortion in electricity supply systems

R. Yacamini, O.A.R. Hamoud, O. Afacan, IEE Proc., Vol. 128, Pt. B, No. 3 , May 1981

[4] An algorithm of the harmonics on traction power system of the electrified railway

Pan Yinxuan, Sun Shugin, Li Chunhua, 1990 IEEE

[5] Frequency domain modelling of traction PWM converters J.A. Taufiq, J. Shen, 1993 The European Power Electronics Association 